\title{
Design and research of WiFi intelligent car based on computer vision technology
}

\author{
Lv Changwu, Qiu Bo \\ Jiangxi Environmental Engineering Vocational College,Ganzhou 341000,China
}

Key words: computer vision; WiFi communication; intelligent car; two-way communication; remote control

\begin{abstract}
In order to realize the work of some dangerous and complex areas, such as the places where the human can not be close to the fire and explosion scene, a kind of intelligent control car with two-way communication ability has put forward in this paper. Based on the machine vision technology and WiFi wireless communication technology, the car can transmit the environmental information of the working area in real time. At the same time, the control terminal can send control commands to the car to realize remote control. The overall framework of the intelligent car is designed, the whole system is composed of six parts, including power supply, control terminal, WiFi communication module, microcontroller, motor and camera, etc.. Finally, the performance of the intelligent car is tested by experiment, and the PCB board of the car control system is made. The experimental results show that the communication distance of communication, WiFi communication over Zigbee and Bluetooth has farther and faster communication speed, can meet the demand of intelligent vehicle two-way real-time communication, which provides a feasible communication scheme for the design of the intelligent car.
\end{abstract}

\section{Introduction}

The full name of Wifi is wireless fidelity, is one of the most widely used wireless network transmission technology. If there is a mobile phone WiFi Internet function, the WiFi signal can not connect to the Internet through places Unicom mobile data, this application has been very extensive, but the application of WiFi in signal control based on little. In the place where there is a WiFi signal, you can not use mobile or Unicom's data connection to the Internet, the application has been very extensive, But the application of WiFi signal in control is still very few. However, compared to the Zigbee wireless transmission and Bluetooth signals with transmission distance, transmission speed and so many advantages, it is used in the design of remote image transmission and control command transmission system, has important significance for improving the transmission rate and transmission distance.

\section{Design of WiFi intelligent car system based on computer vision technology}

The whole system of WiFi intelligent car based on computer vision technology is based on MCU and WiFi module as the core, mainly divided into six parts, including power supply, control terminal, WiFi communication module, microcontroller, motor and camera, the structure of the whole system as shown in figure 1. 


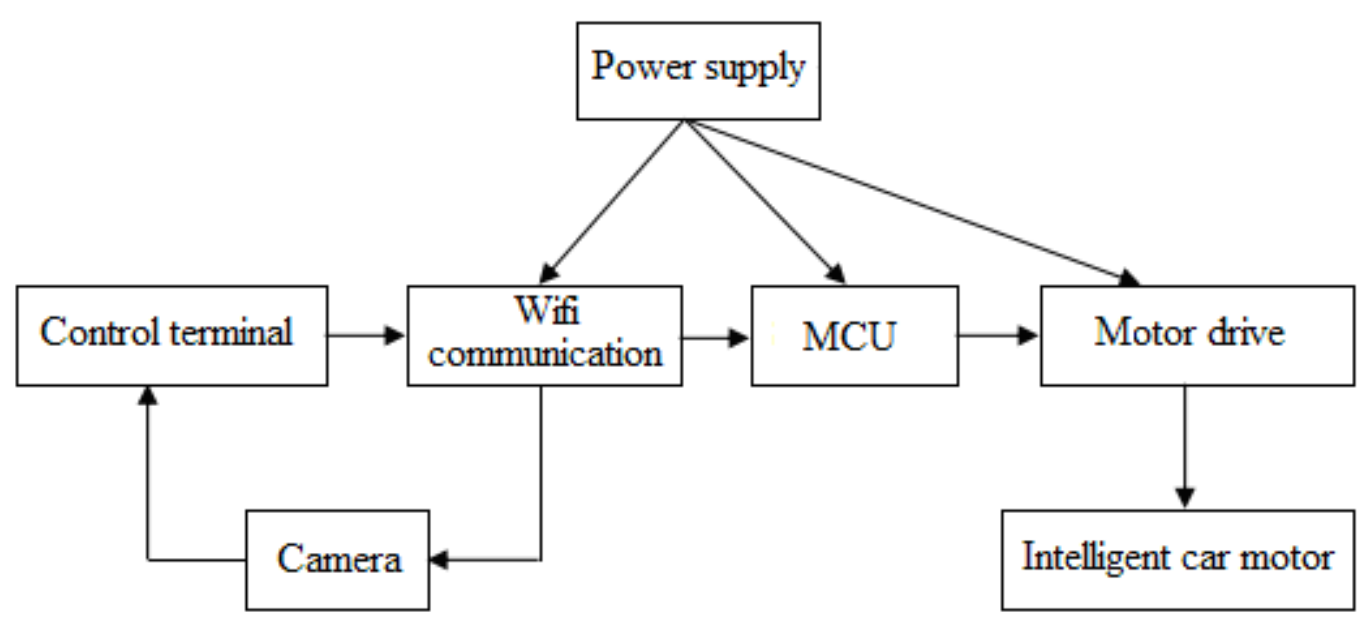

Figure 1 The overall design of the system structure

As shown in Figure 1, the control terminal of WiFi intelligent car control based on computer vision technology can be a computer terminal, and it can also be a mobile phone, the main function of WiFi communication module is responsible for the communication between the control terminal and the lower computer, it can be transmitted back to the real-time image collected by the intelligent car terminal, and can also send control commands to the intelligent car terminal. The system uses intelligent car camera to collect environmental information, then through the MCU and WiFi control module, the real-time image is transmitted back to the host computer, according to the environment information, the host computer can send out the control commands, and the control commands can drive the motor of the intelligent car through the single chip microcomputer to control the moving or turning of the car. The mobile power supply is used in the whole system, and the power supply module can supply power to the WiFi communication module, MCU and motor simultaneously.

\section{Design of Wifi intelligent car motor drive module}

L298N of ST company is used as a motor drive chip, the chip has 15 legs, can drive a two-phase stepper motor or four phase stepper motor, but also can be used to drive two DC motors. the intelligent car based on machine vision uses four DC motors, the drive circuit is shown in Fig. 2.

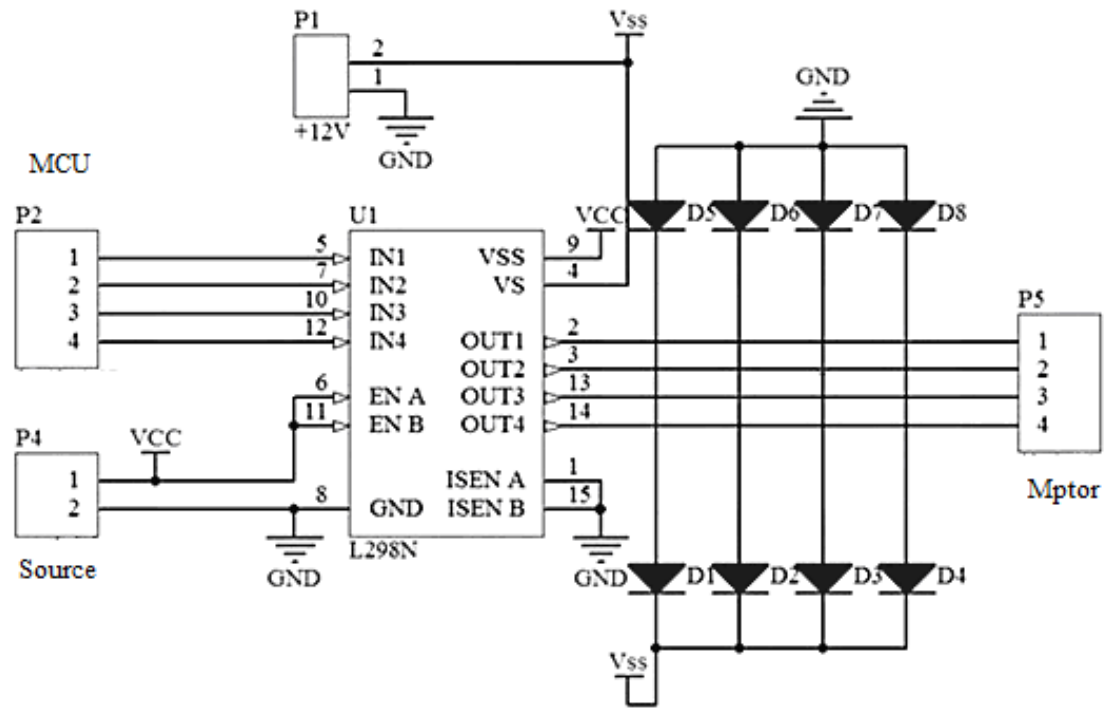

Fig. 2 Schematic circuit driver 
As shown in Fig.2, L298N chip can simultaneously drive two DC motors, assuming that the two motors were M1 and M2, The AB pin is mainly for the motor speed control, speed control using PWM pulse width modulation method. Taking M1 motor as an example, when the IN1 is connected to a low level, IN2 is connected to a high level, you can specify the level when the motor is transferred, instead, the motor rotate reversely, reversing motor can effectively control the car forward backward, the speed and the forward backward control level as shown in table 1.

Table 1 The motor drive status table

\begin{tabular}{|c|c|c|c|c|c|}
\hline \multirow{2}{*}{ Motor } & $\begin{array}{c}\text { Rotation } \\
\text { mode }\end{array}$ & IN1 port & IN2 port & \multicolumn{2}{|c|}{$\begin{array}{r}\text { Input PWM signal change pulse } \\
\text { width adjustable speed }\end{array}$} \\
\cline { 5 - 6 } & Corotation & Low & Hjgh & $/$ & Hjgh \\
\hline \multirow{3}{*}{ M1 } & Reversal & Hjgh & Low & $/$ & Hjgh \\
\cline { 2 - 6 } & Stop & Low & Low & $/$ & Hjgh \\
\hline
\end{tabular}

Table 1 shows the state of the motor driven by the high and low level, the speed can be adjusted by adjusting the speed of the high and low level, the motor forward and backward can be achieved by the motor positive and negative controlling, and through the WiFi real time environment information, operation state by remote control mode intelligent car. Through the environmental information returned by WiFi real-time, remote control can be used to change the operating state of the intelligent car.

\section{Experimental study of WiFi intelligent car based on computer vision technology}

In order to verify the performance of WiFi intelligent vehicle based on computer vision technology, the car communication experiment has been designed, and a PCB board has been produced, board integrated USB interface WiFi communication interface, MCU and WiFi module, the real board of the whole system is shown in Figure 3.

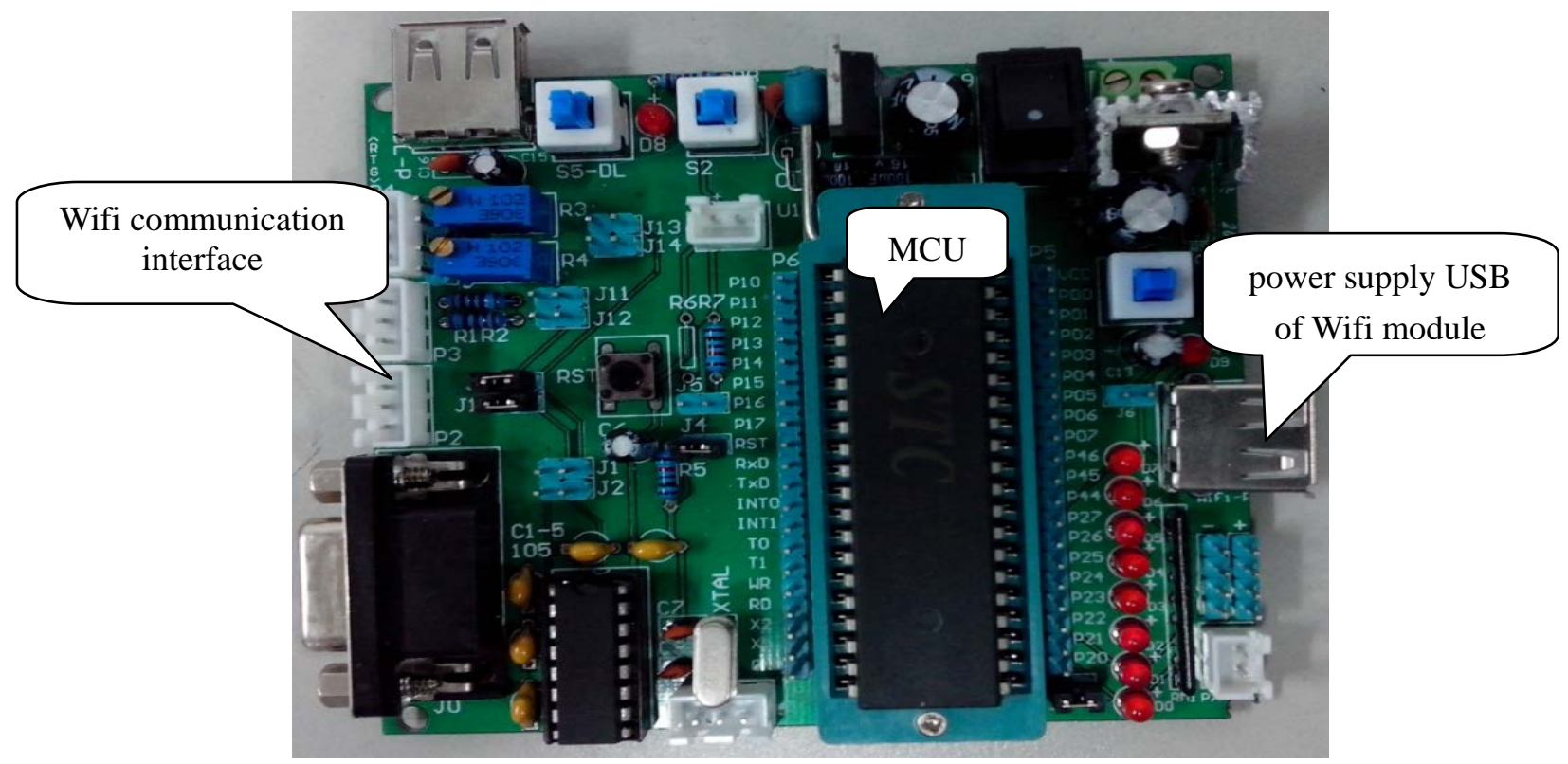

Fig.3 The PCB board of overall system

As shown in Figure 3, after the design of the system, the machine vision was mainly tested in this paper, that is, the transmission of real-time information in WiFi communication environment is tested. In order to verify the superiority of WiFi, we compare it with Bluetooth and Zigbee communication, and get the results as shown in Table 2 . 
Table 2 Comparison of WiFi remote communication effect

\begin{tabular}{|c|c|c|c|}
\hline $\begin{array}{c}\text { Communication } \\
\text { mode }\end{array}$ & $\begin{array}{c}\text { Communica } \\
\text { tion band }\end{array}$ & $\begin{array}{c}\text { Transmission } \\
\text { distance }(\mathrm{m})\end{array}$ & $\begin{array}{c}\text { Transmission } \\
\text { speed }(\mathrm{kb} / \mathrm{s})\end{array}$ \\
\hline Wifi & $3.6 \mathrm{GHz}$ & 160 & 12000 \\
\hline Zigbee & $3.6 \mathrm{GHz}$ & $20-90$ & 350 \\
\hline Bluetooth & $3.6 \mathrm{GHz}$ & 30 & 6000 \\
\hline
\end{tabular}

As shown in Table 2, it is found that, compared with Bluetooth and Zigbee communication technology, WiFi communication technology has longer transmission distance, faster communication speed, and can meet the demand of the real-time image transmission function. When it is used in the design of remote control scheme of the intelligent car, the car intelligent control efficiency can be significantly improved.

\section{Conclusion}

In order to realize the unmanned remote control of dangerous places such as fire and explosion, an intelligent vehicle based on WiFi communication has been designed, and The key design of WiFi communication image real-time transmission module, speed and forward and backward steering control motor drive module are also given. When the intelligent car is in operation, the image can be transmitted back to the working environment in real time, and the control command issued by the control terminal can be received in two directions. In order to verify the visual image transmission ability characteristics of the intelligent car, the intelligent control system of the car and the PCB board of the communication system are made, its communication performance is tested. The test results show that the real-time image transmission can be realized by using $\mathrm{WiFi}$, and the communication distance is long, which can meet the needs of remote video transmission and remote control.

\section{References}

[1] Liu Jinlong, Zheng Zefeng, Ding Weiming, et al. The design and detection of the target spray infrared detector [J]. Jiangsu Agricultural Sciences, Vol.41 (7), (2013), p.368-370

[2] Gao Guoqin, Li Ming. Navigation path recognition of greenhouse mobile robot based on K-means algorithm [J]. Transactions of the Chinese Society of Agricultural Engineering, Vol.30(7), (2014), p.25-33

[3] Yu Genjian. A new organization algorithm in wireless sensor network [J]. Computer Systems \& Applications. Vol.23(2), (2014), p.146-149.

[4] Zhu Daqing, Yan Mingchong. Survey of mobile robot path planning technology[J]. Control and Decision, Vol.25 (7), (2010), p.961-967.

[5] Xia Sheng Sheng, Yan Weisheng. Research on the motion planning of mobile robot based on grid method [J]. Computer Simulation, Vol. 29(12), (2012), p.229-233.

[6] Yu Zhenzhong, Yan Jihong. Improved artificial potential field method for mobile robot path planning [J]. Journal of Harbin Institute of Technology, Vol.43(1), (2011), p.50-55.

[7] Deng Yun, Cheng Xiaohui. Design of wireless sensor network for intelligent irrigation system [J]. Process Automation Instrumentation, Vol.34(2), (2012), p.80-83.

[8] Hu Xiangdong. Research and development of Internet of things [J]. Digital Communication, Vol.37(2), (2010), p.17-21 
[9] Li Peng, Zhang Ruixia, Wang Saichao. Study on atmospheric environment monitoring system based on Internet of things technology [J]. Information \& Communications, No.8, (2013), p.59-60

[10] Li Zhonghao. Design of wireless data acquisition system for oil well based on ZigBee [J]. Electronic Design Engineering. Vol.19(6), (2011), p.110-112

[11] Deng Xin, Zhang Lejun. Overview of survivability enhancement techniques for wireless sensor networks [J]. Transducer and Microsystem Technologies, Vol.33(1), (2014), p.1-10.

[12] Xu Xiongwei, Wang Ping, Xu Shiwu. Research and discussion of synchronization algorithm for wireless sensor networks [J]. Microcontrollers \& Embedded Systems, Vol.12(3), (2012), p.8-11.

[13] Si Haifei, Yang Zhong, Wang Jun. Situation and application of Wireless sensor network research [J]. Journal of Mechanical \& Electrical Engineering, Vol.28(1), (2011), p.16-20.

[14] Xue Ming, Gao Demin. Routing algorithm for maximum lifetime aggregation tree in Wireless Sensor Networks [J]. Transducer and Microsystem Technologies, Vol.33(1), (2014), p.130-133.

[15] Cui Suhui, Chen Guangting,. Repeater placement problem in 3D Wireless Sensor Networks [J]. Journal of Hangzhou Dianzi University, Vol.30(2), (2010), p.81-84. 